\title{
Percepción y hábitos de alimentación durante la cuarentena por COVID-19 en el Perú
}

\author{
Perception and eating habits during the COVID-19 quarantine \\ in Peru
}

Jhoseline Guillen-Sánchez $\square$

${ }^{1}$ Universidad Estadual de Campinas. São Paulo, Brasil

\section{RESUMEN}

Serealizó una encuesta entre mayo y junio de 2020 a 164 personas del departamento de La Libertad, Perú, con el objetivo de comprender y analizar como la pandemia afectó el consumo de alimentos en la población peruana, y si las nuevas formas de hábitos alimenticios pueden ser desfavorables para una alimentación adecuada. El 84,4 \% de los encuestados tenían entre 18 a 35 años y el 53,1 $\%$ eran del sexo femenino. Educación superior técnica era el grado de instrucción predominante. Los cambios más frecuentes se refieren a mayor consumo de frutas y verduras (32\%), y reducción en el consumo productos lácteos como yogurt, leche y queso $(22 \%)$. Productos alimenticios de mediana prioridad, como bebidas industrializadas y productos de confitería, mostraron una reducción de $68 \%$. Posterior a la compra de alimentos, el $45 \%$ de encuestados, higienizan los productos con desinfectantes de uso doméstico. Para el $55 \%$ de encuestados, una alimentación saludable fortalece el sistema inmunológico y reduce la propensión a la infección por coronavirus. Palabras clave: Pandemia, coronavirus, alimentación, Perú.

\section{ABSTRACT}

A survey was conducted between May and June 2020 to 164 people from the department of La Libertad, Peru, with the objective of analyzing how the pandemic affected food consumption in the Peruvian population, and if new forms of eating habits can be unfavorable for adequate nutrition. $84.4 \%$ of those surveyed are between 18 and 35 years old and $53.1 \%$ were female. Technical higher education was the predominant level of instruction. The most frequent changes refer to higher consumption of fruits and vegetables ( $32 \%$ ), and reduction in the consumption of dairy products such as yogurt, milk and cheese (22\%). Medium priority food products, such as industrialized beverages and confectionery products, showed a reduction of $68 \%$. After buying food, $45 \%$ of respondents sanitize the products with disinfectants for domestic use. For $55 \%$ of respondents, a healthy diet strengthens the immune system and reduces the propensity for coronavirus infection. Keywords: Pandemic, coronavirus, food, Peru. 


\section{INTRODUCCIÓN}

En diciembre del 2019, la provincia de Hubei en China, se vio azotado por un agente infeccioso el cual la Organización mundial de la Salud (OMS) identifico como coronavirus 2 (SARS-CoV-2). El virus es transmitido por gotitas respiratorias y contacto físico, una persona infectada puede presentar síntomas como fiebre, tos seca y dificultad al respirar, o puede ser asintomática, esto demuestra la facilidad de esparcirse a nivel mundial (Helmy et al., 2020; Chang et al., 2020). Europa tiene 47 países infectados, principalmente España, Rusia, Reino Unido, Italia y Alemania, mientras que, en latinoamericana, todos los países se han visto afectados, siendo Haití el último en confirmar la presencia de coronavirus en su territorio (BBC News Mundo, 2020).

El once de marzo del 2020, el Perú se declaró en estado de emergencia sanitaria a nivel nacional por COVID-19, mediante decreto de urgencia 008-2020-SA, y el 15 de marzo se dispuso el aislamiento social obligatorio con el acceso a servicios esenciales como el abastecimiento de alimentos, medicinas, agua, energía eléctrica, y otros establecidos en el decreto de urgencia 0442020-PCM (Diario oficial el peruano, 2020).

El pánico colectivo peruano ha conllevado que el abastecimiento de alimentos sea cada vez más difícil y que no exista una racionalización de consumo, debido a esto las personas tienden a aglomerarse en los establecimientos alterando el orden público y suponiendo un mayor riesgo al contagio. Actualmente el Perú cuenta con más de 100 mil infectados por coronavirus, siendo el $40 \%$ mujeres y el $60 \%$ hombres (Ministerio de Salud, 2020).
La alimentación es clave para la existencia humana, sin embargo, puede verse afectada en tiempos de pandemia, por diversos factores como el aislamiento social, los recursos económicos, la ansiedad y el estrés. En un estudio reciente realizado en Argentina, se mostró que el $55 \%$ de la población encuestada ha aumentado su ingesta de alimento, prefiriendo los productos altos en azúcar y grasas saturadas como golosinas y gaseosas (Sudriá, Andreatta, y Defagó, 2020). Una alimentación saludable promueve el fortalecimiento del sistema inmune el cual nos ayuda a combatir infecciones, por lo que es necesario que la alimentación en tiempo de pandemia esté basada en una ingesta mayor de frutas, verduras y legumbres (Flores-Solís, 2020). En este contexto, el objetivo de la investigación fue determinar el impacto del coronavirus sobre los hábitos alimenticios de la población peruana.

\section{MATERIALES Y MÉTODOS}

\section{METODOLOGÍA}

El estudio, transversal y descriptivo, se llevó a cabo en los meses de mayo - julio del 2020 y fue realizado mediante encuesta que releva datos cuantitativos y cualitativos, destinada a personas residentes de distintas provincias del departamento de La Libertad. La encuesta se realizó sobre un formulario digital, con preguntas abiertas y cerradas, que podía completar directamente el entrevistado, o cuando fuera necesario, un encuestador del equipo podía realizar las preguntas y proceder a su rellenado. El instrumento se construyó digitalmente con 24 preguntas categorizadas en dos componentes: sociodemográfico; consumo alimentario y estilos de vida durante la cuarentena por COVID-19. 
El aspecto sociodemográfico, incluyó variables como edad, género, tamaño y composición familiar, y nivel educativo; y en el aspecto percepción y consumo de alimentos, incluye prioridad de compras, higiene e inocuidad en alimentos, consumo y acceso a alimentos; y percepción de hábitos alimenticios. Los parámetros de inclusión a la encuesta correspondieron a personas de 18 y más años, residentes en La Libertad, Perú, que aceptaran participar de la encuesta, mediante un término de consentimiento, y la diligenciaran de forma completa.

Se realizó un muestreo no probabilístico por conveniencia y los individuos fueron escogidos al azar. Cabe resaltar que la metodología de este estudio transversal tuvo limitaciones ya que se llevó a cabo en una muestra de conveniencia reclutada de forma virtual. Sin embargo, la aplicación de una encuesta virtual ha sido la única opción durante la pandemia. La recogida de información de forma virtual limita la participación de los sectores más desfavorecidos y de las personas mayores, mientras que facilita una aceptación mayor en personas con una preocupación mayor por la alimentación y la salud.

\section{Análisis de datos}

Se utilizó estadística descriptiva univariada y medidas de tendencia central apoyadas con el programa estadístico SPSS statistica, para obtener el análisis descriptivo con base en la influencia de los factores sociodemográficos y aspectos alimentarios. La mayoría de variables fueron cualitativasencategorizaciónnominaluordinal.

\section{RESULTADOS Y DISCUSIÓN}

\section{ASPECTOS SOCIALES Y DEMOGRÁ- FICOS}

La muestra total estuvo conformada por 164 individuos, su distribución por sexo indica que el $53,1 \%$ es mujer y el $46,9 \%$ es hombre. El número total de encuestas fue de 164 y la muestra estuvo dividida en $46,9 \%$ varones y 53,1\% mujeres, con edades comprendidas entre los 18 y 60 años, y distribuidos por edades según los siguientes porcentajes: 18 a 35 años: 90,40 \%, y 36 a 60 años: $14,10 \%$. El $60 \%$ reside en zonas urbanas y el resto en rurales. El distrito de Virú tuvo la mayor participación (26,8 \%), le siguen los distritos de Chepén, Paiján y Cascas.

El grado de instrucción, en la mayoría de encuestados era educación superior técnica $(48,9$ $\%$ ), un $18,8 \%$ y $15,6 \%$, era superior universitaria completa e incompleta, respectivamente, y 14,1 \% era educación básica completa. El 53,1 $\%$ tiene un hogar conformado por cuatro miembros como mínimo y el 60,9 \% refiere que tenía un trabajo permanente antes de la pandemia.

\section{Percepción y consumo de alimentos}

Con respecto al consumo de alimentos de primera necesidad, los encuestados respondieron que la cuarentena no afecto su consumo en carnes (20\%), huevos (17\%), verduras (24\%) $\mathrm{y}$, cereales y leguminosas (23\%). Los cambios más frecuentes se refieren a mayor consumo de fruta $(17 \%)$ y verduras $(15 \%)$, y reducción en el consumo de pan (22\%), y productos lácteos como yogurt, leche y queso (22\%). Otros productos alimenticios de mediana prioridad, 
como bebidas industrializadas (gaseosas y jugos), y productos de confitería, mostraron una reducción de consumo, en $31 \%$ y $37 \%$, respectivamente. Estos resultados son similares al estudio realizado por Bejarano-Roncancio et al. (2020) en Colombia, el cual refiere que las durante el confinamiento las familias colombianas aumentaron su consumo en frutas y verduras (63,3\%), y los alimentos que tuvieron menos prioridad fueron, bebidas azucaradas $(50,6 \%)$ y alimentos azucarados $(45,5 \%)$.

El acceso y compra de alimentos de las familias peruanas también se vio afectado durante la cuarentena, el $63 \%$ de los encuestados menciono no ir al supermercado, centro de abastos y/o mercado local por temor al contagio por coronavirus. La compra de productos de panificación en panaderías se redujo en $32 \%$, coincidiendo con la reducción del consumo de pan de los peruanos encuestados. El $45 \%$ de encuestados, higienizan los alimentos y/o envases de éstos, con alcohol, jabón y/o hipoclorito de sodio.

Con respecto al estilo de vida, el $55 \%$ de encuestados menciono que es importante mantener una alimentación saludable que fortalezca el sistema inmunológico, coincidiendo con el aumento en el consumo de frutas y verduras. Sin embargo, el $21 \%$ de los encuestados concuerdan en el limitado poder adquisitivo para comprar alimentos que forman parte de una dieta saludable. La comida industrializada no se muestra como una opción de consumo seguro entre el $32 \%$ de encuestados. Según Moreno-Altamirano (2021), el consumo de alimentos procesados afecta la micro biota intestinal lo cual puede repercutir en el sistema inmunológico y aumentar la predisposición para la COVID-19. Por el contrario, una microbiota intestinal saludable ayuda a prevenir reacciones inmunológicas exageradas (Spector y Gardner, 2020).

La percepción del $24 \%$ de encuestados sobre el consumo de alimento en cuarentena, implica la preocupación de escases de alimentos en el hogar, adicionalmente, el 48,4\% refiere que el tiempo que dedica a su alimentación aumento durante la cuarentena y un $64,1 \%$ que habitualmente cocina una vez, paso a cocinar más veces. Un estudio realizado por Oblitas y Sempertegui (2020), muestra que, durante las semanas de confinamiento, el 43,3\% de personas presento ansiedad, siendo más frecuente en el sexo femenino y en personas mayores de 40 años. Este trastorno puede incrementarse en la persona, cuando hay afectación económica y/o cuando no se realiza actividades lúdico/recreativas. Sin embargo, la ansiedad puede llegar a reducirse en la realización de actividades distractoras, como la preparación de comidas.

La mayoría de los encuestados son trabajadores dependientes e independientes de los sectores prioritarios activos en cuarentena, como sector agrícola y alimentario. Debido a esto, el $62,5 \%$ se encuentra en un parcial aislamiento social. Sin embargo, el $75 \%$ refiere que los ingresos mensuales disminuyeron abismalmente. Esta realidad es percibida en la mayoría de países de América Latina, los países con mayor empleo informal son Bolivia (81 \%), Ecuador (74 \%) y Perú (69\%), según los datos de la Organización Internacional del Trabajo (2010); adicionalmente, aquellos que laboraron durante pandemia tuvieron problemas sociales como dominio del trabajo temporal, sin 
contrato, o plantilla débil (Gamarra-Vílchez, 2021).

\section{CONCLUSIONES}

Los participantes en este estudio refieren cambios alimentarios en el periodo de confinamiento en Perú, con tendencia hacia mayor consumo de alimentos saludables, menor consumo de alimentos de menos interés nutricional y aumento de la práctica de cocinar en casa.

\section{AGRADECIMIENTO}

A los participantes que diligenciaron la encuesta.

\section{REFERENCIAS BIBLIOGRÁFICAS}

Flores-Solís, M.(2020). Nutritional recommendation guides in times of COVID. Dietary recommendations foroutpatients withCOVID-19. Medicina Interna de México, 36 (4), S4-S7.

Helmy, Y., Fawzy, M., Elaswad, A., Sobieh, A., Kenney, S., y Shehata, A. (2020). The COVID-19 Pandemic: A Comprehensive Review of Taxonomy, Genetics, Epidemiology, Diagnosis, Treatment, and Control. Journal of Clinical Medicine, 9, 12-25.

BBC News Mundo. (2020, marzo 30). Coronavirus en América Latina. https://www.bbc. com/mundo/noticias-america-latina-51881075

Ministerio de Salud. (2020). Decreto supremo que declara en emergencia sanitaria a nivel nacional por el plazo de noventa (90) días calendarios y dicta medidas de prevención y control del COVID-19. DECRETO SUPREMO No 0082020-SA. https://cdn.www.gob.pe/uploads/ document/file/605928/DS_008-2020-SA.PDF
Diario Oficial el peruano. (2020). Decreto Supremo que declara Estado de Emergencia Nacional por las graves circunstancias que afectan la vida de la Nación a consecuencia del brote del COVID-19 DECRETO SUPREMO No 0442020-PCM. https://www.gob.pe/institucion/ pcm/normas-legales/460472-044-2020-pcm

Chang, D., Xu, H., Rebaza, A., Sharma, L., y Dela Cruz, C. (2020). Protecting health-care workers from subclinical coronavirusinfection. The Lancet Respiratory Medicine, 8(3), e13. https://doi.org/10.1016/S2213-2600(20)30066-7

World Health Organization. (2020). Mask use in the context of COVID-19. https:// apps.who.int/iris/handle/10665/337199

Sudriá, M., Andreatta, M., y Defagó, M. (2020). Impact of the quarantine by coronaviruses (COVID-19) on food habits in Argentina. DIAETA, 38(171), 10-19.

Bejarano-Roncancio, J., Samacá-Murcia, L., Morales-Salcedo, I., Pava-Cárdenas, L., Cáceres-Jeréz, M., y Durán-Agüero, S. (2020). Caracterización de la seguridad alimentaria en familias colombianas durante el confinamiento por COVID-19. Revista Española de Nutrición Comunitaria, 26(4), 235-241.

Spector, T.D., y Gardner, C.H. (2020). Challenges and opportunities for better nutrition. BMJ (Clinical research ed.), 369, 24-70. https://doi.org/10.1136/bmj.m2470

Oblitas, A., y Sempertegui, N. (2020). Ansiedad en tiempos de aislamiento 
social por COVID-19. Chota, Perú. Avances de enfermería, 38(1), 1-11. https://doi. org/10.15446/av.enferm.v38n1sup1.87589

Oficina Internacional del Trabajo (2009, junio). Para recuperarse de la crisis: Un Pacto Mundial para el Empleo [Conferencia]. 98 ${ }^{\mathrm{a}}$ Conferencia Internacional del Trabajo, Ginebra, Suiza. Gamarra, L. (2021). Entre Escila y Caribdis: pobreza y desempleo post covid-19 en el Perú y el entorno latinoamericano. LABOREM, 24, 471-496. 